\title{
Different Estimation Methods of the Stress-Strength Reliability Restricted Exponentiated Lomax Distribution
}

\author{
Alaa M. Hamad ${ }^{1}$, Bareq B. Salman ${ }^{2 *}$ \\ ${ }^{1}$ Department of Mathematics, Ibn-Al-Haitham College of Education, University of Baghdad, Baghdad 10006, Iraq \\ ${ }^{2}$ The Iraqi Ministry of Education, Rusafa Third Education Directorate, Baghdad 10059, Iraq
}

Corresponding Author Email: bareqbaqe@gmail.com

https://doi.org/10.18280/mmep.080319

Received: 20 October 2020

Accepted: 19 April 2021

\section{Keywords:}

restricted exponentiated Lomax distribution, stress-strength series Rs, moment method, shrinkage estimation

\begin{abstract}
Lomax distribution, a large-scale probabilistic distribution used in industry, economics, actuarial science, queue theory, and Internet traffic modeling, is the most important distribution in reliability theory. In this paper estimating the reliability of Restricted exponentiated Lomax distribution in two cases, when one component $\mathrm{X}$ strength and $\mathrm{Y}$ stress $\mathrm{R}=\mathrm{P}(\mathrm{Y}<\mathrm{X})$, and when system content two component series $R_{S}=\mathrm{P}\left(\mathrm{Y}<\min X_{1}, X_{2}\right)$ when $X_{1}, X_{2}$ strength, Y stress by using different estimation method. such as maximum likelihood, least square and shrinkage methods. A comparison between the outcomes results of the applied methods has been carried out based on mean square error (MSE) to investigate the best method and the obtained results have been displayed via MATLAB software package.
\end{abstract}

\section{INTRODUCTION}

The Lomax distribution and can be called pareto II distribution, if its survival function (SF) is given by where $\beta$, $\gamma$ are, respectively, the scale and shape parameters. Lomax (1954) used distribution to test and interpret data from poor business operations. Abdul-Moniem [1] found a variety of measurement methods for the distribution of Lomax. AlZahrani \& Al-Sobhi [2] investigated the distribution of Lomax through readings resulting from correct censorship. Jha \& Chakraborty [3] and Chaves Agudelo [4] respectively related to the distributional properties and continuity of the Lumax norm values. Yu and Brazauskas [5] referred to and submitted applications for this position in support of data resulting from inaccurate and incorrectly truncated Lomax distributions. Chakraborty [6]. describe the distribution of Lomax as a blending distribution of Poisson modulus and obtain a discreet distribution of Poisson-Lomax. Lemonte and Cordeiro [7] propose Bayesian's approximation of the survival function of the Lomax distribution.

This application has been widely used in many fields, such as the disparity in the amount of income and resources of people and nations, the disparity in urban environments, actuarial sciences, engineering and medical sciences, and computer science engineering and modelling and its efficiency. Model data from official wealth and income institutions Tarko [8], Rahman and Aslam [9], data from the delivery of control devices and computers on computer servers Abdullahi and Ieren [10], durability and life-testing Hassan et al. [11], Characteristics of ROC receivers were used.

Al-Marzouki [12] and El-Bassiouny et al. [13] worked on several modifications to this distribution, like Piossin Lomax distribution, Beta Lomax distribution, gamma Lomax distribution, Weibull Lomax distribution, Power Lomax distribution, exponentiated Lomax distribution. The main aim of this paper is to study restricted exponentialized distribution of Lomax. Reliability is the likelihood that the system can continue in operation for a given period of time, stress-strength reliability can be analysed by two models, the first stressstrength (S-S) model in one component $\mathrm{R}=\mathrm{P}(\mathrm{Y}<\mathrm{X})$ when $\mathrm{Y}$ stress- $\mathrm{X}$ is high, the second model in system content in two component sequence $\mathrm{R}_{\mathrm{S}}=\mathrm{P}\left(\mathrm{Y}<\min \mathrm{X}_{1}, \mathrm{X}_{2}\right)$ when $\mathrm{X}_{1}, \mathrm{X}_{2}$ strength and $Y$ stress [14]. The p.d.f for Exponentiated Lomax distribution [15].

\section{METHODOLOGY}

Let $x$ be random variable from power distribution with shape parameter $(\alpha, \theta)$ and scale parameter $(\lambda)$; then the probability density function $(p d f)$ for Exponentiated Lomax distribution is defined as follow [4]

$$
\begin{aligned}
& f(x, \alpha, \theta, \lambda)= \\
& \alpha \theta \lambda\left[1-(1+\lambda x)^{-\theta}\right]^{\alpha-1}(1+\lambda x)^{-(\theta+1)}, \mathrm{x}>0 \quad \theta, \alpha, \beta>0
\end{aligned}
$$

The Restricted exponentiated Lomax distribution when $\theta=1$, $\lambda=1$.

The probability density function for restricted exponentiated Lomax distribution

$$
f(x, \alpha)=\alpha\left[1-(1+x)^{-1}\right]^{\alpha-1}(1+x)^{-2}, \mathrm{x}>0
$$

The c.d.f for restricted exponentiated Lomax distribution

$$
F(x, \alpha)=\left[1-(1+x)^{-1}\right]^{\alpha} \mathrm{x}>0
$$


The reliability for restricted exponentiated Lomax distribution

$$
R(x, \alpha)=1-\left[1-(1+x)^{-1}\right]^{\alpha} \mathrm{x}>0
$$

\section{THE RELIABILITY ESTIMATION}

\subsection{The reliability for one component stress-strength model [14]}

Let $X \sim R \operatorname{Lom}\left(x, \alpha_{1}\right)$ and $Y \sim R \operatorname{Lom}\left(y, \alpha_{2}\right), \mathrm{X}$ arise strength and $\mathrm{y}$ arise stress.

Than

$$
\begin{gathered}
R=P(y<x) \\
R=\int_{0}^{\infty x} \int_{0} f(x) f(y) d y d x \\
=\int_{0}^{\infty} \int_{0}^{x} \alpha_{1}\left[1-(1+x)^{-1}\right]^{\alpha_{1}-1}(1+x)^{-2} \\
\alpha_{2}\left[1-(1+y)^{-1}\right]^{\alpha_{2}-1}(1+y)^{-2} d y d x \\
=\int_{0}^{\infty} \alpha_{1}\left[1-(1+x)^{-1}\right]^{\alpha_{1}-1}(1+x)^{-2} \\
\left.\left[\left[1-(1+y)^{-1}\right]^{\alpha_{2}}\right]_{0}^{x}\right] d x \\
=\int_{0}^{\infty} \alpha_{1}\left[1-(1+x)^{-1}\right]^{\alpha_{1}+\alpha_{2}-1}(1+x)^{-2} d x \\
R=\frac{\alpha_{1}}{\alpha_{1}+\alpha_{2}}
\end{gathered}
$$

\subsection{The reliability for two component series stress-strength} model $[16,17]$

Assume $X_{1}, X_{2}$ arises strength have (RLom) with shape parameters $\alpha_{1}, \alpha_{2}$ and $\mathrm{Y}$ is stress have (RLom) with shape parameter $\alpha_{3}$

$$
\begin{aligned}
& Z=\min \left(x_{1}, x_{2}\right) \\
& R_{S}=P(\mathrm{y}<z)=\int_{0}^{\infty} \int_{0}^{z} f(y) f(z) d y d z \\
& R_{S}=\int_{0}^{\infty} F_{y}(\mathrm{z}) \mathrm{f}(\mathrm{z}) \mathrm{dz} \\
& F_{Z}(\mathrm{z})=p(Z<z)=1-P(Z>z) \\
& F_{z}(z)=1-p(\min x>z) \\
& F_{z}(z)=1-p\left(x_{1}>z\right) p\left(x_{2}>z\right) \\
& F_{z}(\mathrm{z})=1-p\left(x_{1}>z\right) p\left(x_{2}>z\right) \\
& F_{z}(\mathrm{z})= \\
& 1-\left[1-\left(1-(1+z)^{-1}\right)^{\alpha}\right]\left[1-\left(1-(1+z)^{-1}\right)^{\alpha}{ }^{\alpha}\right] \\
& F_{z}(\mathrm{z})=\left[1-(1+z)^{-1}\right]^{\alpha_{1}}+\left[1-(1+z)^{-1}\right]^{\alpha_{2}} \\
& -\left[1-(1+z)^{-1}\right]^{\alpha_{1}+\alpha_{2}}
\end{aligned}
$$




$$
\hat{\alpha}_{1 m l e}=\frac{-n}{\sum_{i=1}^{n} \operatorname{Ln}\left[1-\left(1+x_{i}\right)^{-1}\right]}
$$

And by derive function for $\alpha_{2}, \alpha_{3}$ and equate the equations with zero, we get

$$
\begin{aligned}
& \hat{\alpha}_{2 m l e}=\frac{-m}{\sum_{j=1}^{m} \operatorname{Ln}\left[1-\left(1+y_{j}\right)^{-1}\right]} \\
& \hat{\alpha}_{3 m l e}=\frac{-w}{\sum_{q=1}^{w} \operatorname{Ln}\left[1-\left(1+z_{q}\right)^{-1}\right]}
\end{aligned}
$$

For one component reliability in maximum likelihood, put Eq. (10) and (11) in Eq. (8)

$$
\hat{R}_{m l e}=\frac{\alpha_{1 m l e}}{\alpha_{1 m l e}+\alpha_{2 m l e}}
$$

For two component system reliability in maximum likelihood, put Eq. (10), (11) and (12) in Eq. (9)

$$
\begin{aligned}
& \hat{R}_{s_{\text {mle }}}=\frac{\alpha_{1 m l e}}{\alpha_{1 m l e}+\alpha_{3 m l e}}+\frac{\alpha_{2 m l e}}{\alpha_{2 m l e}+\alpha_{3 m l e}} \\
& -\frac{\alpha_{1 m l e}+\alpha_{2 m l e}}{\alpha_{1 m l e}+\alpha_{2 m l e}+\alpha_{3 m l e}}
\end{aligned}
$$

\subsection{Least square method $[17,18]$}

The least square method is often used in mathematics problems and the estimation of parameter, estimation of reliability, and reliability engineering.

The (c.d.f) for Resected exponentiated Lomax distribution

$$
\begin{aligned}
& F\left(x_{i}\right)=\left[1-\left(1+x_{i}\right)^{-1}\right]^{\alpha_{1}} \\
& {\left[F\left(x_{i}\right)\right]^{\frac{1}{\alpha_{1}}}=1-\left(1+x_{i}\right)^{-1}}
\end{aligned}
$$

Taking $L n$ for the equation

$$
\begin{gathered}
\operatorname{Ln}\left[F\left(x_{i}\right)\right]^{\frac{1}{\alpha_{1}}}=\operatorname{Ln}\left[1-\left(1+x_{i}\right)^{-1}\right] \\
\frac{1}{\alpha_{1}} \operatorname{Ln}\left[F\left(x_{i}\right)\right]=\operatorname{Ln}\left[1-\left(1+x_{i}\right)^{-1}\right] \\
a x+b=y_{i} \\
a=\frac{1}{\alpha_{1}} \\
b=0 \\
x_{i}=\operatorname{Ln}\left[F\left(x_{i}\right)\right] \\
y_{i}=\operatorname{Ln}\left[1-\left(1+x_{i}\right)^{-1}\right]
\end{gathered}
$$

$$
\begin{gathered}
a=\frac{\sum_{i=1}^{n} x_{i} y_{i}-\frac{\sum_{i=1}^{n} x_{i} \sum_{i=1}^{n} y_{i}}{n}}{\sum_{i=1}^{n} x_{i}^{2}-\frac{\left(\sum_{i=1}^{n} x_{i}\right)^{2}}{n}} \\
\hat{\alpha}_{1 / s}=\frac{\sum_{i=1}^{n}\left[\operatorname{Ln} F\left(x_{i}\right)\right]^{2}-\frac{\left[\sum_{i=1}^{n} \operatorname{Ln} F\left(x_{i}\right)\right]^{2}}{n}}{\operatorname{LnF}\left(x_{i}\right) \operatorname{Ln}\left[1-\left(1+x_{i}\right)^{-1}\right]} \\
\sum_{i=1}^{n}-\frac{\sum_{i=1}^{n} \operatorname{Ln} F\left(x_{i}\right) \sum_{i=1}^{n} \operatorname{Ln}\left[1-\left(1+x_{i}\right)^{-1}\right]}{n}
\end{gathered}
$$

From (c.d.f) for $\mathrm{Y}$ and $\mathrm{Z}$

$$
\begin{aligned}
& \hat{\alpha}_{2 l s}=\frac{\sum_{j=1}^{m}\left[\operatorname{LnF}\left(y_{j}\right)\right]^{2}-\frac{\left[\sum_{j=1}^{m} \operatorname{Ln} F\left(y_{j}\right)\right]^{2}}{m}}{\operatorname{Ln} F\left(y_{j}\right) \operatorname{Ln}\left[1-\left(1+y_{j}\right)^{-1}\right]} \\
& \sum_{j=1}^{m}-\frac{\sum_{j=1}^{m} \operatorname{LnF}\left(y_{j}\right) \sum_{j=1}^{m} \operatorname{Ln}\left[1-\left(1+y_{j}\right)^{-1}\right]}{m} \\
& \hat{\alpha}_{3 l s}=\frac{\sum_{q=1}^{w}\left[\operatorname{Ln} F\left(z_{q}\right)\right]^{2}-\frac{\left[\sum_{q=1}^{w} \operatorname{Ln} F\left(z_{q}\right)\right]^{2}}{w}}{\operatorname{Ln} F\left(z_{q}\right) \operatorname{Ln}\left[1-\left(1+z_{q}\right)^{-1}\right]} \\
& \sum_{q=1}^{w}-\frac{\sum_{q=1}^{w} \operatorname{LnF}\left(z_{q}\right) \sum_{q=1}^{w} \operatorname{Ln}\left[1-\left(1+z_{q}\right)^{-1}\right]}{w}
\end{aligned}
$$

For one component reliability in Least square, put Eq. (15) and (16) in Eq. (8)

$$
\hat{R}_{L s}=\frac{\alpha_{1 L s}}{\alpha_{1 L s}+\alpha_{2 L s}}
$$

For two component system reliability in Least square, put Eq. (15), (16) and (17) in Eq. (9)

$$
\widehat{R}_{s_{L s}}=\frac{\alpha_{1 L s}}{\alpha_{1 L s}+\alpha_{3 L s}}+\frac{\alpha_{2 L s}}{\alpha_{2 L s}+\alpha_{3 L s}}-\frac{\alpha_{1 L s}+\alpha_{2 L s}}{\alpha_{1 L s}+\alpha_{2 L s}+\alpha_{3 L s}}
$$

\subsection{Shrinkage estimation methods $[14,17]$}

Is one of the important methods, Thompson presented the equation for this method

$$
\hat{\alpha}_{s h}=\phi(\hat{\alpha}) \hat{\alpha}_{u b}+(1-\phi(\hat{\alpha})) \alpha_{0}, 0<\phi(\hat{\alpha})<1
$$


It is one of the Bayesian methods, Thompson stated it is possible to be a function of weight function or constant.

To find $\alpha_{u b}$

$\hat{\alpha}_{m l e}==\frac{-n}{\sum_{i=1}^{n} \operatorname{Ln}\left[1-\left(1+x_{i}\right)^{-1}\right]}$ is biased

$$
\begin{gathered}
E\left(\hat{\alpha}_{m l e}\right)=\frac{n}{n-1} \alpha \\
\hat{\alpha}_{u b}=\frac{n-1}{n} \times \hat{\alpha}_{m l e} \\
=\frac{-n}{\sum_{i=1}^{n} \operatorname{Ln}\left[1-\left(1+x_{i}\right)^{-1}\right]} \times \frac{(n-1)}{n} \\
\hat{\alpha}_{1 u b}=\frac{-(n-1)}{\sum_{i=1}^{n} \operatorname{Ln}\left[1-\left(1+x_{i}\right)^{-1}\right]}
\end{gathered}
$$

(1) Shrinkage weight factor $\left(S h_{1}\right)[14,17]$

In this subsection we considered $\phi_{1}(\hat{\alpha})=\left|\frac{\sin n}{n}\right|$, put in Thompson equation, we get

$$
\hat{\alpha}_{1 s h_{1}}=\left|\frac{\sin \mathrm{n}}{n}\right| \hat{\alpha}_{1 u b}+\left(1-\left|\frac{\sin \mathrm{n}}{n}\right|\right) \alpha_{0}
$$

By $\phi_{2}(\hat{\alpha}), \phi_{2}(\hat{\alpha})$ found

$$
\begin{aligned}
& \hat{\alpha}_{2 s h_{1}}=\left|\frac{\sin \mathrm{m}}{m}\right| \hat{\alpha}_{2 u b}+\left(1-\left|\frac{\sin \mathrm{m}}{m}\right|\right) \alpha_{0} \\
& \hat{\alpha}_{3 s h_{1}}=\left|\frac{\sin \mathrm{w}}{w}\right| \hat{\alpha}_{3 u b}+\left(1-\left|\frac{\sin \mathrm{w}}{w}\right|\right) \alpha_{0}
\end{aligned}
$$

For one component reliability in shrinkage weight factor, put Eq. (22) and (23) in Eq. (8)

$$
\hat{R}_{s h 1}=\frac{\alpha_{1 s h 1}}{\alpha_{1 s h 1}+\alpha_{2 s h 1}}
$$

For two component system reliability in shrinkage weight factor, put Eq. (22), (23) and (24) in Eq. (9)

$$
\hat{R}_{s_{s h 1}}=\frac{\alpha_{1 s h 1}}{\alpha_{1 s h 1}+\alpha_{3 s h 1}}+\frac{\alpha_{2 s h 1}}{\alpha_{2 s h 1}+\alpha_{3 s h 1}}-\frac{\alpha_{1 s h 1}+\alpha_{2 s h 1}}{\alpha_{1 s h 1}+\alpha_{2 s h 1}+\alpha_{3 s h 1}}
$$

(2) Constant Shrinkage Function $S h_{2}[14,17]$

In this subsection we considered $\phi(\hat{\alpha})=k$ the suggested case, put in Thompson equation, we get

$$
\hat{\alpha}_{1 s h_{2}}=k_{1} \widehat{\alpha}_{1 u b}+\left(1-k_{1}\right) \alpha_{0}
$$

By $\phi_{2}(\hat{\alpha}), \phi_{2}(\hat{\alpha})$ found

$$
\begin{aligned}
& \hat{\alpha}_{2 s h_{2}}=k_{2} \widehat{\alpha}_{2 u b}+\left(1-k_{2}\right) \alpha_{0} \\
& \hat{\alpha}_{3 s h_{2}}=k_{3} \widehat{\alpha}_{3 u b}+\left(1-k_{3}\right) \alpha_{0}
\end{aligned}
$$

For one component reliability in constant shrinkage function, put Eq. (27) and (28) in Eq. (8)

$$
\hat{R}_{s h 2}=\frac{\alpha_{1 s h 2}}{\alpha_{1 s h 2}+\alpha_{2 s h 2}}
$$

For two component system reliability in constant shrinkage function, put Eq. (27), (28) and (29) in Eq. (9)

$$
\begin{aligned}
& \hat{R}_{s_{s h 2}}= \\
& \frac{\alpha_{1 s h 2}}{\alpha_{1 s h 2}+\alpha_{3 s h 2}}+\frac{\alpha_{2 s h 2}}{\alpha_{2 s h 2}+\alpha_{3 s h 2}}-\frac{\alpha_{1 s h 2}+\alpha_{2 s h 2}}{\alpha_{1 s h 2}+\alpha_{2 s h 2}+\alpha_{3 s h 2}}
\end{aligned}
$$

(3) Beta Shrinkage Function $S h_{3}[14,17]$

In this subsection we considered $\phi_{1}(\hat{\alpha})=B(1, n)$, put in Thompson equation, we get

$$
\hat{\alpha}_{1 s h_{3}}=B(1, n) \hat{\alpha}_{1 u b}+(1-B(1, n)) \alpha_{0}
$$

By $\phi_{2}(\hat{\alpha}), \phi_{2}(\hat{\alpha})$ found

$$
\begin{aligned}
& \hat{\alpha}_{2 s h_{3}}=B(1, m) \hat{\alpha}_{2 u b}+(1-B(1, m)) \alpha_{0} \\
& \hat{\alpha}_{3 s h_{3}}=B(1, \mathrm{w}) \hat{\alpha}_{3 u b}+(1-B(1, \mathrm{w})) \alpha_{0}
\end{aligned}
$$

For one component reliability in constant shrinkage function, put Eq. (32) and (33) in Eq. (8)

$$
\widehat{R}_{s h 3}=\frac{\alpha_{1 s h 3}}{\alpha_{1 s h 3}+\alpha_{2 s h 3}}
$$

For two component system reliability in constant shrinkage function, put Eqns. (32), (33) and (34) in Eq. (9)

$$
\widehat{R}_{s_{s h 3}}=\frac{\alpha_{1 s h 3}}{\alpha_{1 s h 3}+\alpha_{3 s h 3}}+\frac{\alpha_{2 s h 3}}{\alpha_{2 s h 3}+\alpha_{3 s h 3}}-\frac{\alpha_{1 s h 3}+\alpha_{2 s h 3}}{\alpha_{1 s h 3}+\alpha_{2 s h 3}+\alpha_{3 s h 3}}
$$

\section{THE SIMULATION}

Simulation is solving problems by building a model similar to reality. The simulation with using unlike sample size $(20,30$, $50,100)$ and built on 1000 replications.

Step1: $\mathrm{x}$ according to the uniform distribution on interval $(0,1)$ as $r_{1}, r_{2}, \ldots \ldots \ldots \ldots r_{n}, y$ according to the uniform distribution on interval $(0,1)$ as $e_{1} e_{2} \ldots \ldots \ldots \ldots \ldots e_{m}$ and $\mathrm{z}$ according to the uniform distribution on interval $(0,1)$ as $f_{1}, f_{2}, \ldots \ldots \ldots \ldots f_{w}$.

Step2: from $F(x, \alpha)=\left[1-(1+x)^{-1}\right]^{\alpha}$,

$$
x_{i}=\left[1-\left(\mathrm{r}_{i}\right)^{\frac{1}{\alpha_{1}}}\right]^{-1}-1, \quad \mathrm{i}=1,2,3 \ldots \ldots \ldots . \mathrm{n}
$$

And by the same way

$$
y_{j}=\left[1-\left(\mathrm{e}_{j}\right)^{\frac{1}{\alpha_{2}}}\right]^{-1}-1, \mathrm{j}=1,2,3 \ldots \ldots \ldots . . \mathrm{m}
$$




$$
z_{q}=\left[1-\left(\mathrm{f}_{q}\right)^{\frac{1}{\alpha_{3}}}\right]^{-1}-1, \quad \mathrm{q}=1,2,3 \ldots \ldots \ldots \mathrm{w}
$$

Step3: calculated $\hat{\alpha}_{1 m l e}, \hat{\alpha}_{2 m l e}$ and $\hat{\alpha}_{3 m l e}$ by Eqns. (10), (11) and (12).

Step4: calculated $\hat{\alpha}_{1 L s}, \hat{\alpha}_{2 L s}$ and $\hat{\alpha}_{3 L s}$ by Eqns. (15), (16) and (17).

Step5: calculated $\hat{\alpha}_{1 s h i}, \hat{\alpha}_{2 s h i}$ and $\hat{\alpha}_{3 s h i}$ for $i=1,2,3$ by equations (22),(23),(24), (27),(28),(29), (32),(33) and (34).

Step6: calculated $\hat{R}_{m l e}, \widehat{R}_{L s}, \widehat{R}_{s h 1}, \widehat{R}_{s h 2}$ and $\hat{R}_{s h 3}$ by Eqns. (13), (18), (25), (30) and (35).

Step7: calculated $\hat{R}_{s(m l e)}, \hat{R}_{s(L s)}, \hat{R}_{s(s h 1)}, \hat{R}_{s(s h 2)}$ and $\hat{R}_{s(s h 3)}$ by Eqns. (14), (19), (26), (31) and (36).

Step8: calculated MSE for the purpose of comparing methods $M S E=\frac{\sum_{i=1}^{L}\left(\widehat{R}_{i}-R\right)^{2}}{L}$

We using random sample for $\mathrm{R},(\mathrm{n}, \mathrm{m})=(20,50),(20,100)$, $(30,20),(30,30),(30,100),(50,30),(50,100),(100,20)$ and $(100,100)$, the random sample for $R_{s},(\mathrm{n}, \mathrm{m}, \mathrm{w})=(20,30,50)$, $(20,50,100),(30,20,50),(30,20,100),(50,20,30),(50,30,50)$, $(100,50,20),(100,30,100)$

\section{NUMERICAL RESULTS}

(1) As a consequence, for practical work and taking the mean square error as the indicator of preference between the different estimator methods, and through the Tables 1-12 shown, the following result obtained: -

(i) Small sample size $(\mathrm{n}=20,30)$

when $\mathrm{n}, \mathrm{m}, \mathrm{w}=20,30,50,100$ the minimum mean square error (MSE) for the (S-S) reliability estimators of stress-strength model of Restricted exponentiated Lomax distribution in two cases, is holds using the shrinkage estimator based on shrinkage weight each $(\alpha 1, \alpha 2, \alpha 3)$ this result indicates that, the shrinkage (sh2) estimator method of (S-S) reliability was given the best results. then this methods Sh1, Sh3, MLE, LS, and MOM respectively.

(ii) Medium sample size $(\mathrm{n}=50)$

when $\mathrm{n}, \mathrm{m}, \mathrm{w}=20,30,50,100$ the minimum mean square error (MSE) for the (S-S) reliability estimators of stress-strength model of Restricted exponentiated Lomax distribution in two cases, is holds using the shrinkage estimator based on shrinkage weight each $(\alpha 1, \alpha 2, \alpha 3)$ this result indicates that, the shrinkage ( $s h 2)$ estimator method of (S-S) reliability was given the best results. then this methods Sh1, Sh3, MLE, LS, and MOM respectively.

(iii) Large sample size $(\mathrm{n}=100)$

when $\mathrm{n}=100$, and $\mathrm{n}, \mathrm{m}, \mathrm{w}=20,30,50,100$ the minimum mean square error (MSE) for the (S-S) reliability estimators of stress-strength model of Restricted exponentiated Lomax distribution in two cases, is holds using the shrinkage estimator based on shrinkage weight each $(\alpha 1, \alpha 2, \alpha 3)$ this result indicates that, the shrinkage ( $\operatorname{sh} 2)$ estimator method of (S-S) reliability was given the best results. then this methods Sh1, Sh3, MLE, LS, and MOM respectively.

Table 1. Estimation for restricted exponentiated Lomax distribution when $\mathrm{R}=0.50000$, alpha1 $=1$, alpha $=1$

\begin{tabular}{cccccc}
\hline$(\mathrm{n}, \mathrm{m})$ & $\widehat{\boldsymbol{R}}_{\text {mle }}$ & $\widehat{\boldsymbol{R}}_{\text {sh1 }}$ & $\widehat{\boldsymbol{R}}_{\text {sh2 }}$ & $\widehat{\boldsymbol{R}}_{\text {sh3 }}$ & $\widehat{\boldsymbol{R}}_{\text {Ls }}$ \\
\hline$(20,50)$ & 0.500018 & 0.500043 & 0.500000 & 0.500027 & 0.500088 \\
$(20,100)$ & 0.500232 & 0.500039 & 0.500001 & 0.500032 & 0.497263 \\
$(30,20)$ & 0.500186 & 0.500061 & 0.500001 & 0.500055 & 0.500098 \\
$(30,30)$ & 0.500943 & 0.500033 & 0.499999 & 0.499841 & 0.500610 \\
$(30,100)$ & 0.504399 & 0.500013 & 0.500001 & 0.500008 & 0.502921 \\
$(50,30)$ & 0.500165 & 0.500024 & 0.500003 & 0.500007 & 0.507453 \\
$(50,100)$ & 0.523003 & 0.500092 & 0.499992 & 0.500204 & 0.502468 \\
$(100,20)$ & 0.502265 & 0.500063 & 0.499997 & 0.500077 & 0.500552 \\
$(100,100)$ & 0.505683 & 0.500008 & 0.500002 & 0.500003 & 0.497369 \\
\hline
\end{tabular}

Table 2. MSE values for restricted exponentiated Lomax distribution when $\mathrm{R}=0.50000$, alpha $1=1$, alpha $2=1$

\begin{tabular}{ccccccc}
\hline$(\mathrm{n}, \mathrm{m})$ & $\boldsymbol{m s}_{\text {mle }}$ & $\boldsymbol{m s}_{\text {sh1 }}$ & $\boldsymbol{m s}_{\text {sh2 }}$ & $\boldsymbol{m s e}_{\text {sh3 }}$ & $\boldsymbol{m s}_{\text {Ls }}$ & Best \\
\hline$(20,50)$ & 0.006004571 & 0.000005321 & 0.000000001 & 0.000006819 & 0.006930884 & $s h_{2}$ \\
$(20,100)$ & 0.001931677 & 0.000000603 & 0.000000002 & 0.000000474 & 0.001501423 & $s h_{2}$ \\
$(30,20)$ & 0.079365647 & 0.000018942 & 0.000000008 & 0.000020651 & 0.020862330 & $s h_{2}$ \\
$(30,30)$ & 0.004954503 & 0.000003416 & 0.000000021 & 0.000002716 & 0.007504992 & $s h_{2}$ \\
$(30,100)$ & 0.034846743 & 0.000000761 & 0.000000011 & 0.000013074 & 0.027662564 & $s h_{2}$ \\
$(50,30)$ & 0.002636406 & 0.000000431 & 0.000000062 & 0.000000942 & 0.001977947 & $s h_{2}$ \\
$(50,100)$ & 0.011340570 & 0.000002109 & 0.000000071 & 0.000002119 & 0.003873458 & $s h_{2}$ \\
$(100,20)$ & 0.003521091 & 0.000000106 & 0.000000007 & 0.000007894 & 0.028396555 & $s h_{2}$ \\
$(100,100)$ & 0.0079074274 & 0.000000191 & 0.000000007 & 0.000000746 & 0.01995959 & $s h_{2}$ \\
\hline
\end{tabular}

Table 3. Estimation for restricted exponentiated Lomax distribution when $\mathrm{R}=0.3333$, alpha1 $=1$, alpha $2=2$

\begin{tabular}{cccccc}
\hline$(\mathrm{n}, \mathrm{m})$ & $\widehat{\boldsymbol{R}}_{\text {mle }}$ & $\widehat{\boldsymbol{R}}_{\text {sh1 }}$ & $\widehat{\boldsymbol{R}}_{\text {sh2 }}$ & $\widehat{\boldsymbol{R}}_{\text {sh3 }}$ & $\widehat{\boldsymbol{R}}_{\text {Ls }}$ \\
\hline$(20,50)$ & 0.335631 & 0.333365 & 0.333332 & 0.333316 & 0.325567 \\
$(20,100)$ & 0.327187 & 0.333342 & 0.333338 & 0.333398 & 0.327214 \\
$(30,20)$ & 0.324752 & 0.333399 & 0.333337 & 0.333362 & 0.330789 \\
$(30,30)$ & 0.335689 & 0.333316 & 0.333332 & 0.333317 & 0.330132 \\
$(30,100)$ & 0.327376 & 0.333332 & 0.333330 & 0.333230 & 0.327603 \\
$(50,30)$ & 0.331616 & 0.333353 & 0.333337 & 0.333326 & 0.332716 \\
$(50,100)$ & 0.342981 & 0.333459 & 0.333336 & 0.333388 & 0.334939 \\
$(100,20)$ & 0.339614 & 0.333348 & 0.333336 & 0.333390 & 0.334438 \\
$(100,100)$ & 0.330841 & 0.333239 & 0.333338 & 0.333323 & 0.330372 \\
\hline
\end{tabular}


Table 4. MSE values for restricted exponentiated Lomax distribution when $\mathrm{R}=0.33333$, alpha $1=1$, alpha $2=2$

\begin{tabular}{ccccccc}
\hline$(\mathrm{n}, \mathrm{m})$ & $\boldsymbol{m s}_{\mathrm{mle}}$ & $\boldsymbol{m s}_{\text {sh1 }}$ & $\boldsymbol{m s e}_{\text {sh2 }}$ & $\boldsymbol{m s e}_{\text {sh3 }}$ & $\boldsymbol{m s}_{\text {Ls }}$ & Best \\
\hline$(20,50)$ & 0.005352361 & 0.000003602 & 0.000000002 & 0.000005564 & 0.02980766 & $s h_{2}$ \\
$(20,100)$ & 0.003178918 & 0.000000956 & 0.0000000010 & 0.000000297 & 0.00487209 & $s h_{2}$ \\
$(30,20)$ & 0.08773482 & 0.00001897 & 0.000000023 & 0.000016782 & 0.02459109 & $s h_{2}$ \\
$(30,30)$ & 0.013253287 & 0.000006602 & 0.000000009 & 0.000006980 & 0.03352395 & $s h_{2}$ \\
$(30,100)$ & 0.00072002 & 0.000000028 & 0.000000001 & 0.000000067 & 0.000523096 & $s h_{2}$ \\
$(50,30)$ & 0.006439432 & 0.000000317 & 0.000000002 & 0.000007540 & 0.02756774 & $s h_{2}$ \\
$(50,100)$ & 0.01044502 & 0.000003903 & 0.000000001 & 0.000003903 & 0.002244443 & $s h_{2}$ \\
$(100,20)$ & 0.051442106 & 0.000008906 & 0.000000017 & 0.000007096 & 0.012955439 & $s h_{2}$ \\
$(100,100)$ & 0.009869049 & 0.000000397 & 0.000000001 & 0.000000798 & 0.021795632 & $s h_{2}$ \\
\hline
\end{tabular}

Table 5. Estimation for restricted exponentiated Lomax distribution when $\mathrm{R}=0.500000$, alpha $1=2$, alpha $2=2$

\begin{tabular}{cccccc}
\hline$(\mathrm{n}, \mathrm{m})$ & $\widehat{\boldsymbol{R}}_{\text {mle }}$ & $\widehat{\boldsymbol{R}}_{\text {sh1 }}$ & $\widehat{\boldsymbol{R}}_{\text {sh2 }}$ & $\widehat{\boldsymbol{R}}_{\text {sh3 }}$ & $\widehat{\boldsymbol{R}}_{\text {Ls }}$ \\
\hline$(20,50)$ & 0.500316 & 0.500038 & 0.500000 & 0.500078 & 0.496542 \\
$(20,100)$ & 0.496684 & 0.500032 & 0.500001 & 0.500088 & 0.497520 \\
$(30,20)$ & 0.489659 & 0.499965 & 0.499998 & 0.499931 & 0.499541 \\
$(30,30)$ & 0.504431 & 0.500028 & 0.499999 & 0.500009 & 0.500231 \\
$(30,100)$ & 0.505652 & 0.500065 & 0.500005 & 0.500214 & 0.504843 \\
$(50,30)$ & 0.493614 & 0.499985 & 0.499997 & 0.499936 & 0.494731 \\
$(50,100)$ & 0.506671 & 0.499988 & 0.499997 & 0.499966 & 0.502878 \\
$(100,20)$ & 0.500665 & 0.499972 & 0.499996 & 0.499918 & 0.496983 \\
$(100,100)$ & 0.497690 & 0.499989 & 0.499997 & 0.499988 & 0.496810 \\
\hline
\end{tabular}

Table 6. MSE values for restricted exponentiated Lomax distribution when $\mathrm{R}=0.500000$, alpha $1=2$, alpha $2=2$

\begin{tabular}{ccccccc}
\hline$(\mathrm{n}, \mathrm{m})$ & $\boldsymbol{m s e}_{\mathrm{mle}}$ & $\boldsymbol{m s e}_{\mathrm{sh} 1}$ & $\boldsymbol{m s}_{\mathrm{sh} 2}$ & $\boldsymbol{m s e}_{\mathrm{sh} 3}$ & $\boldsymbol{m s e}_{\text {Ls }}$ & Best \\
\hline$(20,50)$ & 0.006002888 & 0.000006653 & 0.000000005 & 0.000006387 & 0.006936754 & $s h_{2}$ \\
$(20,100)$ & 0.001903431 & 0.000000893 & 0.0000000005 & 0.000000892 & 0.001503238 & $s h_{2}$ \\
$(30,20)$ & 0.012999982 & 0.000006790 & 0.000000001 & 0.000002287 & 0.003711212 & $s h_{2}$ \\
$(30,30)$ & 0.003123431 & 0.000000983 & 0.0000000008 & 0.000000700 & 0.002820478 & $s h_{2}$ \\
$(30,100)$ & 0.005445089 & 0.000000932 & 0.000000004 & 0.000002001 & 0.004306543 & $s h_{2}$ \\
$(50,30)$ & 0.004426541 & 0.000000077 & 0.000000001 & 0.000000679 & 0.005231317 & $s h_{2}$ \\
$(50,100)$ & 0.005700240 & 0.000002344 & 0.000000001 & 0.000002390 & 0.002825643 & $s h_{2}$ \\
$(100,20)$ & 0.001053541 & 0.000000065 & 0.000000001 & 0.000000788 & 0.003149832 & $s h_{2}$ \\
$(100,100)$ & 0.001229834 & 0.000000018 & 0.000000001 & 0.000000211 & 0.001721086 & $s h_{2}$ \\
\hline
\end{tabular}

Table 7. Estimation for restricted exponentiated Lomax distribution when $R_{S}=0.166666$, alpha1 $=1$, alpha $2=1$, alpha3 $=2$

\begin{tabular}{cccccc}
\hline$(\mathrm{n}, \mathrm{m}, \mathrm{w})$ & $\widehat{\boldsymbol{R}}_{\text {mle }}$ & $\widehat{\boldsymbol{R}}_{\text {sh1 }}$ & $\widehat{\boldsymbol{R}}_{\text {sh2 }}$ & $\widehat{\boldsymbol{R}}_{\text {sh3 }}$ & $\widehat{\boldsymbol{R}}_{\text {Ls }}$ \\
\hline$(20,30,50)$ & 0.1709856 & 0.16667656 & 0.1666655 & 0.1666703 & 0.1574295 \\
$(20,50,100)$ & 0.1691818 & 0.16667653 & 0.1666623 & 0.1666747 & 0.1600361 \\
$(30,20,50)$ & 0.1685698 & 0.1666522 & 0.1666653 & 0.1666622 & 0.1597813 \\
$(30,20,100)$ & 0.1685690 & 0.1666614 & 0.1666677 & 0.1666330 & 0.1577180 \\
$(50,20,30)$ & 0.1687850 & 0.1666453 & 0.1666659 & 0.1666689 & 0.1602754 \\
$(50,30,50)$ & 0.1685688 & 0.1666689 & 0.1666670 & 0.1666802 & 0.1608074 \\
$(100,50,20)$ & 0.1688880 & 0.1666343 & 0.1666666 & 0.1666386 & 0.1606704 \\
$(100,30,100)$ & 0.1686943 & 0.1666652 & 0.1666661 & 0.1666804 & 0.1609259 \\
\hline
\end{tabular}

Table 8. MSE values for restricted exponentiated Lomax distribution when $R_{s}=0.166666$, alpha1=1, alpha2 $=1$, alpha3 $=2$

\begin{tabular}{|c|c|c|c|c|c|c|}
\hline$(\mathrm{n}, \mathrm{m}, \mathrm{w})$ & $\boldsymbol{m s e}_{\mathrm{mle}}$ & $\boldsymbol{m s e}_{\mathrm{sh} 1}$ & $\boldsymbol{m s e}_{\mathrm{sh} 2}$ & $\boldsymbol{m s e}_{\mathrm{sh} 3}$ & $\boldsymbol{m s e}_{\mathrm{Ls}}$ & Best \\
\hline$(20,30,50)$ & 0.0006174 & 0.00000067634 & 0.00000000054 & 0.00000068433 & 59742 & $s h_{2}$ \\
\hline$(20$ & & & & & & $s h_{2}$ \\
\hline & 420 & 3899 & 0.00000000019 & 0.0000 & $0.000^{\top}$ & $s h_{2}$ \\
\hline$(30,20,100)$ & 0.0005 & $0 \Omega 000$ & 0.0000 & 0.0000 & 0.0 & $s h_{2}$ \\
\hline & 0.0003 & 0.000000 & 0.00000000039 & 0.00000016 & 0.0006895476 & $s h_{2}$ \\
\hline & & & 0.00 & 0.000 & 0.00 & $s h_{2}$ \\
\hline (1) & 2 & 5 & 000 & 0.000 & 0.00 & $s h_{2}$ \\
\hline$(100,30,100)$ & 0.00030140523 & 0.00000000711 & 0.00000000018 & 0.00000009305 & 0.00061853114 & $s h_{2}$ \\
\hline
\end{tabular}

Table 9. Estimation for restricted exponentiated Lomax distribution when $R_{s}=0.2333333$, alpha1 $=1$, alpha $2=2$, alph $3=2$

\begin{tabular}{cccccc}
\hline$(\mathrm{n}, \mathrm{m}, \mathrm{w})$ & $\widehat{\boldsymbol{R}}_{\mathrm{mle}}$ & $\widehat{\boldsymbol{R}}_{\text {sh1 }}$ & $\widehat{\boldsymbol{R}}_{\text {sh2 }}$ & $\widehat{\boldsymbol{R}}_{\text {sh3 }}$ & $\widehat{\boldsymbol{R}}_{\mathrm{Ls}}$ \\
\hline$(20,30,50)$ & 0.2375786 & 0.2333875 & 0.2333339 & 0.2333430 & 0.22176534 \\
$(20,50,100)$ & 0.2378754 & 0.2333764 & 0.2333338 & 0.2333764 & 0.2257634 \\
$(30,20,50)$ & 0.2352988 & 0.2332984 & 0.2333331 & 0.2332899 & 0.2237634 \\
\hline
\end{tabular}




\begin{tabular}{cccccc}
\hline$(\mathrm{n}, \mathrm{m}, \mathrm{w})$ & $\widehat{\boldsymbol{R}}_{\text {mle }}$ & $\widehat{\boldsymbol{R}}_{\text {sh1 }}$ & $\widehat{\boldsymbol{R}}_{\text {sh2 }}$ & $\widehat{\boldsymbol{R}}_{\text {sh3 }}$ & $\widehat{\boldsymbol{R}}_{\text {Ls }}$ \\
\hline$(30,20,100)$ & 0.2364456 & 0.2333334 & 0.2333333 & 0.2333763 & 0.2236558 \\
$(50,20,30)$ & 0.2356763 & 0.2333397 & 0.2333332 & 0.2333401 & 0.22507534 \\
$(50,30,50)$ & 0.2360976 & 0.2333365 & 0.2333339 & 0.2333398 & 0.2267390 \\
$(100,50,20)$ & 0.2345856 & 0.2333270 & 0.2333317 & 0.2333378 & 0.22493407 \\
$(100,30,100)$ & 0.2353054 & 0.2333359 & 0.2333339 & 0.2333378 & 0.2263209 \\
\hline
\end{tabular}

Table 10. MSE values for restricted exponentiated Lomax distribution when $R_{S}=0.2333333$, alpha1=1, alpha $2=2$, alph $3=2$

\begin{tabular}{ccccccc}
\hline$(\mathrm{n}, \mathrm{m}, \mathrm{w})$ & $\boldsymbol{m s e}_{\mathrm{mle}}$ & $\boldsymbol{m s e}_{\text {sh1 }}$ & $\boldsymbol{m s e}_{\text {sh2 }}$ & $\boldsymbol{m s e}_{\text {sh3 }}$ & $\boldsymbol{m s e}_{\text {Ls }}$ & Best \\
\hline$(20,30,50)$ & 0.00102086543 & 0.00000117840 & 0.00000000568 & 0.00000116795 & 0.0018048939 & $s h_{2}$ \\
$(20,50,100)$ & 0.00088799563 & 0.00000087894 & 0.00000000112 & 0.00000090654 & 0.0015655437 & $s h_{2}$ \\
$(30,20,50)$ & 0.00077767654 & 0.00000076794 & 0.00000000101 & 0.00000078644 & 0.0015388943 & $s h_{2}$ \\
$(30,20,100)$ & 0.00064908605 & 0.00000035893 & 0.00000000018 & 0.00000044789 & 0.0012449763 & $s h_{2}$ \\
$(50,20,30)$ & 0.00058566742 & 0.00000002874 & 0.00000000062 & 0.00000022087 & 0.0011069276 & $s h_{2}$ \\
$(50,30,50)$ & 0.00049668763 & 0.00000001548 & 0.00000000049 & 0.00000016954 & 0.0009695334 & $s h_{2}$ \\
$(100,50,20)$ & 0.00044985634 & 0.00000031974 & 0.00000000051 & 0.00000033901 & 0.0009549564 & $s h_{2}$ \\
$(100,30,100)$ & 0.0003777564 & 0.00000002003 & 0.00000000022 & 0.00000009023 & 0.0007064435 & $s h_{2}$ \\
\hline
\end{tabular}

Table 11. Estimation for restricted exponentiated Lomax distribution when $R_{s}=0.333333$, alpha $1=2$, alpha $2=2$, alpha3 $=2$

\begin{tabular}{cccccc}
\hline$(\mathrm{n}, \mathrm{m}, \mathrm{w})$ & $\widehat{\boldsymbol{R}}_{\text {mle }}$ & $\widehat{\boldsymbol{R}}_{\text {sh1 }}$ & $\widehat{\boldsymbol{R}}_{\text {sh2 }}$ & $\widehat{\boldsymbol{R}}_{\text {sh3 }}$ & $\widehat{\boldsymbol{R}}_{\text {Ls }}$ \\
\hline$(20,30,50)$ & 0.3358653 & 0.3338943 & 0.3333334 & 0.3332764 & 0.3140397 \\
$(20,50,100)$ & 0.3356539 & 0.3333845 & 0.3333331 & 0.3333765 & 0.3189897 \\
$(30,20,50)$ & 0.3356222 & 0.3333601 & 0.3333339 & 0.3333633 & 0.3218444 \\
$(30,20,100)$ & 0.3375908 & 0.3333654 & 0.3333351 & 0.3333602 & 0.3201345 \\
$(50,20,30)$ & 0.3357989 & 0.3333389 & 0.3333332 & 0.3333349 & 0.3218432 \\
$(50,30,50)$ & 0.3353458 & 0.3333327 & 0.3333355 & 0.3333654 & 0.3254357 \\
$(100,50,20)$ & 0.3366432 & 0.3333574 & 0.3333313 & 0.3333722 & 0.3222308 \\
$(100,30,100)$ & 0.3361147 & 0.3333503 & 0.3333347 & 0.3333618 & 0.3249873 \\
\hline
\end{tabular}

Table 12. MSE values for restricted exponentiated Lomax distribution when $R_{s}=0.333333$, alpha1 $=2$, alpha $2=2$, alpha3 $=2$

\begin{tabular}{ccccccc}
\hline$(\mathrm{n}, \mathrm{m}, \mathrm{w})$ & $\boldsymbol{m s}_{\mathrm{mle}}$ & $\boldsymbol{m s e}_{\text {sh1 }}$ & $\boldsymbol{m s}_{\text {sh2 }}$ & $\boldsymbol{m s e}_{\mathrm{sh} 3}$ & $\boldsymbol{m s e}_{\mathrm{Ls}}$ & $\mathrm{Best}$ \\
\hline$(20,30,50)$ & 0.001317654 & 0.0000015190 & 0.0000000012 & 0.0000015765 & 0.0026700653 & $s h_{2}$ \\
$(20,50,100)$ & 0.0010534432 & 0.0000007896 & 0.0000000034 & 0.0000009134 & 0.0020323643 & $s h_{2}$ \\
$(30,20,50)$ & 0.0009149653 & 0.0000008652 & 0.0000000003 & 0.0000008874 & 0.0017894529 & $s h_{2}$ \\
$(30,20,100)$ & 0.0010415532 & 0.0000007945 & 0.0000000099 & $\mathbf{0 . 0 0 0 0 0 0 9 9 4 4}$ & 0.0020258834 & $s h_{2}$ \\
$(50,20,30)$ & 0.0008262490 & 0.0000000564 & 0.0000000007 & 0.0000003833 & 0.0016046097 & $s h_{2}$ \\
$(50,30,50)$ & 0.0005669300 & 0.0000000866 & 0.0000000008 & 0.0000001698 & 0.0011088342 & $s h_{2}$ \\
$(100,50,20)$ & 0.0009200763 & 0.0000008565 & 0.0000000066 & 0.0000008621 & 0.0017188000 & $s h_{2}$ \\
$(100,30,100)$ & 0.0006110646 & 0.0000000172 & 0.0000000006 & 0.0000001976 & 0.0012104456 & $s h_{2}$ \\
\hline
\end{tabular}

(2) The result of simulation for the (S-S) reliability estimators of stress-strength model of Restricted exponentiated Lomax distribution for studied methods for different sample size $(n=20,30,50,100)$. From tables it is clear that, the shrinkage (constant weight factor $\left(s h_{2}\right)$ ) estimates is the best in performance for each case. Then his first shrinkage way $\left(s h_{1}\right)$ comes after. From the tables for each $(n)$, and each $(\alpha 1, \alpha 2, \alpha 3)$ we conclude that, the suggested Shrinkage method have good performance based on constant - weight factor $\left(s h_{2}\right)$ has minimum mean square error (MSE) in at the majority of cases.

\section{CONCLUSION}

According to the result of the simulation process, we have revealed the following: shrinkage method (constant give that the shrinkage function $s h_{2}$ ), can be considered as the best method for estimation reliability content one component $\mathrm{R}$ and reliability system $R_{s}$, for all cases with sample size $\mathrm{n}=20,30,50,100, \mathrm{~m}=20,30,50,100$ in compare with all other applied method in this work has the smallest mean square error (MSE).

\section{REFERENCES}

[1] Abdul-Moniem, I.B. (2012). Recurrence relations for moments of lower generalized order statistics from exponentiated Lomax distribution and its characterization. J. Math. Comput. Sci., 2(4): 999-1011.

[2] Al-Zahrani, B., Al-Sobhi, M. (2013). On parameters estimation of Lomax distribution under general progressive censoring. Journal of Quality and Reliability Engineering. http://dx.doi.org/10.1155/2013/431541

[3] Jha, P., Chakraborty, A. (2014). Post-fordism, Global Production Networks and Implications for Labour. Institute for Studies in Industrial Development.

[4] Chaves Agudelo, J.M. (2016). (Re) meanings of nature in a neoliberal globalized modern world: Three cases of Colombian indigenous ethnicities (Pijao, Cofan and Muisca-Chibcha) (Doctoral dissertation). http://hdl.handle.net/11343/129357.

[5] Yu, D., Brazauskas, V. (2017). Model uncertainty in operational risk modeling due to data truncation: A single $\begin{array}{llll}\text { risk } & \text { case. } & \text { Risks, } & \end{array}$ https://doi.org/10.3390/risks5030049

[6] Chakraborty, S. (2015). Generating discrete analogues of continuous probability distributions-a survey of methods 
and constructions. Journal of Statistical Distributions and Applications, 2(1): 1-30.

[7] Lemonte, A.J., Cordeiro, G.M. (2013). An extended Lomax distribution. Statistics, 47(4): 800-816. https://doi.org/10.1080/02331888.2011.568119

[8] Tarko, A.P. (2018). Estimating the expected number of crashes with traffic conflicts and the Lomax Distribution-A theoretical and numerical exploration. Accident Analysis \& Prevention, 113: 63-73. https://doi.org/10.1016/j.aap.2018.01.008

[9] Rahman, J., Aslam, M. (2017). On estimation of twocomponent mixture inverse Lomax model via Bayesian approach. International Journal of System Assurance Engineering and Management, 8(1): 99-109.

[10] Abdullahi, U.K., Ieren, T.G. (2018). On the inferences and applications of transmuted exponential Lomax distribution. International Journal of Advanced Probability and Statistics, 6(1): 30-36.

[11] Hassan, A.S., Assar, S.M., Shelbaia, A. (2016). Optimum step-stress accelerated life test plan for Lomax distribution with an adaptive type-II Progressive hybrid censoring. Journal of Advances in Mathematics and Computer Science,

$1-19$. https://doi.org/10.9734/10.9734/BJMCS/2016/21964

[12] Al-Marzouki, S. (2018). A new generalization of power Lomax distribution. Int. J. Math. Appl, 7: 59-68.

[13] El-Bassiouny, A.H., Abdo, N.F., Shahen, H.S. (2015).
Exponential Lomax distribution. International Journal of Computer Applications, 121(13): https://doi.org/10.5120/21602-4713

[14] Sail, F., Salman, A.N. (2017). On reliability estimation of stress-strength model. Master thesis college of education Ibn AL-Haitham University of Baghdad.

[15] Tahir, M.H., Cordeiro, G.M., Mansoor, M., Zubair, M. (2015). The Weibull-Lomax distribution: properties and applications. Hacettepe Journal of Mathematics and Statistics, 44(2): 461-480.

[16] Manuel, B.T., Gerardo, D.M. (2012). Stress/ Strength Models to Estimation Systems Reliability $R(t)=P(x<y)$. International Journal of Engineering Research, 7(3).

[17] Salman, B.B., Alaa, M.H., Adel, A.H. (2019). On reliability estimation of stress-strength(s-s) modified exponentiated Lomax distribution. Journal of Mechanics of Continua and Mathematical Sciences, 14(4): 387-405. https://doi.org/10.26782/jmcms.2019.08.00032

[18] Akhter, A.S. (2013). Methods for estimating the parameters of the power function distribution. Pakistan Journal of Statistics and Operation Research, 213-224. https://doi.org/10.18187/pjsor.v9i2.488

[19] Hamad, A.M. (2017). Estimation of the parameter of an exponential distribution when applying maximum likelihood and probability plot methods using simulation. Ibn Al-Haitham Journal for Pure and Applied Science, 25(1). 